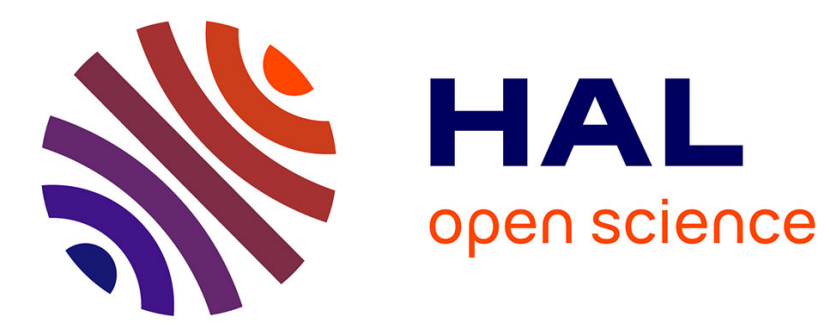

\title{
Coherent and incoherent ultrasound backscatter from cell aggregates
}

Romain de Monchy, François Destrempes, Ratan Saha, Guy Cloutier, Emilie Franceschini

\section{- To cite this version:}

Romain de Monchy, François Destrempes, Ratan Saha, Guy Cloutier, Emilie Franceschini. Coherent and incoherent ultrasound backscatter from cell aggregates. Journal of the Acoustical Society of America, 2016, 140 (3), pp.2173-2184. 10.1121/1.4962502 . hal-01783903

\section{HAL Id: hal-01783903 \\ https://hal.science/hal-01783903}

Submitted on 2 May 2018

HAL is a multi-disciplinary open access archive for the deposit and dissemination of scientific research documents, whether they are published or not. The documents may come from teaching and research institutions in France or abroad, or from public or private research centers.
L'archive ouverte pluridisciplinaire HAL, est destinée au dépôt et à la diffusion de documents scientifiques de niveau recherche, publiés ou non, émanant des établissements d'enseignement et de recherche français ou étrangers, des laboratoires publics ou privés. 


\title{
Coherent and incoherent ultrasound backscatter from cell aggregates
}

\author{
Romain de Monchy \\ Laboratoire de Mécanique et d'Acoustique, Aix-Marseille Université, CNRS UPR 7051, Centrale Marseille, \\ 4 impasse Nikola TESLA, CS 40006, 13453 Marseille cedex 13, France
}

François Destrempes

Laboratory of Biorheology and Medical Ultrasonics, University of Montreal Hospital Research Centre (CRCHUM), 900 St-Denis (suite R11.720), Montreal, Quebec, Canada, H2X 0A9

Ratan K. Saha

Department of Applied Sciences, Indian Institute of Information Technology Allahabad, Devghat, Jhalwa, Allahabad 210112, India

Guy Cloutier*

Laboratory of Biorheology and Medical Ultrasonics, University of Montreal Hospital Research Centre (CRCHUM), 900 St-Denis (suite R11.720), Montreal, Quebec, H2X 0A9, Canada

Emilie Franceschini ${ }^{\dagger}$

Laboratoire de Mécanique et d'Acoustique LMA CNRS UPR 7051, Aix-Marseille Université, Centrale Marseille, 4 impasse Nikola TESLA, CS 40006, 13453 Marseille cedex 13, France

(Dated: May 3, 2017)

\begin{abstract}
The Effective Medium Theory (EMT) combined with the Structure Factor Model was recently developed to model the ultrasound backscatter from aggregating Red Blood Cells (RBCs) [Franceschini, Metzger, Cloutier, IEEE UFFC, 2011]. The EMT assumes that aggregates can be treated as homogeneous effective spheres and the structure factor considers the interactions between the effective spheres. In this study, the EMT is further developed to decompose the differential backscattering cross section of a single cell aggregate into coherent and incoherent components. The coherent component corresponds to the average backscatter from the effective scatterer, and the incoherent component considers the fluctuation of the scattering wave around its average within the effective scatterer. A new theoretical expression for the incoherent component based on the structure factor is proposed and compared with another formulation based on the Gaussian direct correlation function. This theoretical improvement is assessed using computer simulations of ultrasound backscatter from aggregating cells. The consideration of the incoherent component based on the structure factor allows to approximate the simulations satisfactorily for a $k r_{\text {ag }}$ limit

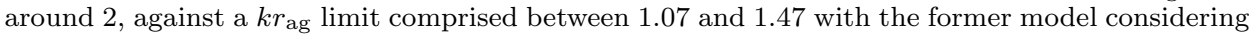
only the coherent component.
\end{abstract}

PACS numbers: 43.80.Cs, 43.80.Qf, 43.35.Bf

Keywords: quantitative ultrasound, structure factor, aggregate, effective medium theory, ultrasound tissue characterization

\section{INTRODUCTION}

Ultrasound scattering from biological tissues exhibits a frequency dependence which is related to the structure of the insonified medium. For determining the tissue microstructure, one approach consists in fitting the measured frequency-dependent backscatter coefficient (BSC) to a theoretical BSC derived using an appropriate theoretical scattering model. Two theoretical scattering models are often used in the field of quantitative ultrasound

\footnotetext{
*Also at: Department of Radiology, Radio-Oncology and Nuclear Medicine, and Institute of Biomedical Engineering, University of Montreal, Montreal, Quebec, H3T IJ4, Canada

${ }^{\dagger} \mathrm{E}$-mail: franceschini@lma.cnrs-mrs.fr
}

(QUS) imaging. The first model, named the spherical Gaussian model, describes a tissue as a random inhomogeneous continuum with impedance fluctuations. ${ }^{1}$ The second model considers tissues as randomly distributed discrete scatterers with an impedance differing from a homogeneous background medium. ${ }^{2}$ In both models, the scatterers are assumed to be independently and uniformly randomly distributed, which may correspond to the case of a low scatterer concentration. However, biological tissues often have high cellular concentrations and complex structures that invalidate the assumption of those models. ${ }^{3,4}$ For example, some tumors have densely packed cells and eventually aggregating cells (such as breast sarcoma ${ }^{5}$ ); and blood contains a high volume fraction of red blood cells (between 30 and 50\%) that form aggregates having rouleaux structures in normal blood 
or clumps in some pathologies, as in diabetes mellitus. ${ }^{6,7}$ Therefore, theoretical scattering models have been proposed to include high cellular concentration together with aggregating cells, and to estimate scatterer structures. ${ }^{8,9}$ A scattering model called the Effective Medium Theory combined with the Structure Factor Model (EMTSFM) was recently proposed..$^{10}$ In this model, the aggregates of cells are viewed as individual scatterers which have effective properties determined by the acoustical characteristics and concentration of cells within aggregates. The approximation of cell aggregates as homogeneous effective scatterers is combined with the structure factor model to consider the concentrated medium; i.e., to consider the interference effects caused by the correlations between the spatial positions of effective scatterers. The EMTSFM allows characterization of the radius and of the compactness of cell aggregates, as shown in a previous simulation study. ${ }^{11}$

The goal of the current study was to further develop the EMTSFM and to extend its validity into a larger frequency range. In that aim, the Effective Medium Theory (EMT) was modified to decompose the differential backscattering cross section of a single cell aggregate into coherent and incoherent components as proposed by Morse and Ingard. ${ }^{12}$ The coherent component corresponds to the average wave emerging from the effective scatterer, and the incoherent component represents the fluctuation of the scattering wave around its average within the effective scatterer. Note that only the coherent component was taken into account in our previous works. ${ }^{10,11} \mathrm{~A}$ new theoretical expression for the incoherent component based on the structure factor is proposed. This theoretical improvement was assessed using three dimensional (3D) computer simulations of ultrasound backscatter from aggregating cells. The new incoherent component based on the structure factor was compared to the incoherent component based on a Gaussian direct correlation function proposed by Morse and Ingard. ${ }^{12}$

\section{THEORY}

In this section, the theoretical expression for the ultrasound backscattering response from one aggregate of cells is proposed. Then, the theoretical BSC expression for an ensemble of identical aggregates that was developed in Ref. 10 is briefly recalled.

\section{A. Differential backscattering cross-section $\sigma_{\text {ag }}$ of one aggregate of cells}

In the sequel, we consider identical cells distributed randomly inside an aggregate. It is assumed that the incident wavelength is large compared to the cell size. Consequently, the cell shape can be approximated by a sphere of radius $a$ having an equivalent volume $V_{\mathrm{c}}=\frac{4}{3} \pi a^{3}$. Cells are described in terms of their mass density $\rho_{c}$ and compressibility $\kappa_{c}$, and their surrounding medium is characterized by its mass density $\rho_{0}$ and compressibility $\kappa_{0}$. An aggregate of cells, denoted $\mathbf{V}_{\mathrm{ag}}$, is assumed to be spherical as it occurs in some tissues (e.g., red blood cell aggregates in pathological cases ${ }^{6,7}$ or breast sarcoma tumors ${ }^{5}$ ).

The EMT model assumes that an aggregate of cells can be treated as an effective homogeneous spherical scatterer of radius $r_{\mathrm{ag}}$. As previously studied by Morse and Ingard, ${ }^{12}$ the differential backscattering cross-section $\sigma_{\mathrm{ag}}$ of an aggregate can be decomposed into two components:

$$
\sigma_{\mathrm{ag}}=\sigma_{\mathrm{ag}, \mathrm{coh}}+\sigma_{\mathrm{ag}, \mathrm{inc}},
$$

where the coherent component $\sigma_{\text {ag,coh }}$ corresponds to the average wave emerging from the effective scatterer, and the incoherent component $\sigma_{\text {ag,inc }}$ describes the fluctuation of the scattering wave around its average within the effective scatterer, as detailed below.

\section{The scattering amplitude}

Using Born and far-field approximations, the backscattered wave pressure is expressed in terms of the backscattered amplitude $\Phi_{\text {ag }} ;$ i.e., $p_{\mathrm{s}}(\mathbf{r})=\left(e^{i k r} / r\right) \Phi_{\mathrm{ag}}(k)$, with ${ }^{13}$

$$
\Phi_{\text {ag }}(k)=\frac{k^{2}}{4 \pi} \int_{\mathbf{V}_{\text {ag }}}\left[\gamma_{\kappa}\left(\mathbf{r}_{0}\right)-\gamma_{\rho}\left(\mathbf{r}_{0}\right)\right] e^{2 i k \mathbf{n}_{0} \cdot \mathbf{r}_{0}} d^{3} \mathbf{r}_{0},
$$

where $k$ is the wavenumber, $\mathbf{r}_{0}$ is the position in the three dimensional space, $\mathbf{n}_{0}$ is the incident wave direction, $\gamma_{\kappa}\left(\mathbf{r}_{\mathbf{0}}\right)=\left(\kappa\left(\mathbf{r}_{\mathbf{0}}\right)-\kappa_{0}\right) / \kappa_{0}$ and $\gamma_{\rho}\left(\mathbf{r}_{\mathbf{0}}\right)=\left(\rho\left(\mathbf{r}_{\mathbf{0}}\right)-\rho_{0}\right) / \rho\left(\mathbf{r}_{\mathbf{0}}\right)$ are the fractional variations in compressibility and mass density, respectively. One has

$$
\gamma_{\kappa}\left(\mathbf{r}_{\mathbf{0}}\right)-\gamma_{\rho}\left(\mathbf{r}_{\mathbf{0}}\right)=\left\{\begin{array}{cl}
\gamma_{\kappa}-\gamma_{\rho} & \left(\text { if } \mathbf{r}_{\mathbf{0}}\right. \text { is inside the cells) } \\
0 & \left(\text { if } \mathbf{r}_{\mathbf{0}} \text { is outside the cells }\right),
\end{array}\right.
$$

where $\gamma_{\kappa}=\left(\kappa_{c}-\kappa_{0}\right) / \kappa_{0}$ and $\gamma_{\rho}=\left(\rho_{c}-\rho_{0}\right) / \rho_{c}$. Assuming $N$ identical cells inside an aggregate with centers located at positions $\mathbf{r}_{j}, j=1, \ldots, N$, and considering the change of variable $\mathbf{r}_{0}^{\prime}=\mathbf{r}_{0}-\mathbf{r}_{j}$, the scattering amplitude can be expressed as

$$
\Phi_{\mathrm{ag}}(k)=\frac{k^{2}\left(\gamma_{\kappa}-\gamma_{\rho}\right)}{4 \pi} V_{c}\left(V_{c}^{-1} \int_{\mathbf{V}_{a}} e^{2 i k \mathbf{n}_{0} \cdot \mathbf{r}_{0}^{\prime}} d^{3} \mathbf{r}_{0}^{\prime}\right) \sum_{j=1}^{N} e^{2 i k \mathbf{n}_{0} \cdot \mathbf{r}_{j}},
$$

where $\mathbf{V}_{a}$ is a sphere of radius $a$ centered at the origin (see Appendix A for details). The integral term in Eq. (3) is equal to

$V_{c}^{-1} \int_{\mathbf{V}_{a}} e^{2 i k \mathbf{n}_{0} \cdot \mathbf{r}_{0}^{\prime}} d^{3} \mathbf{r}_{0}^{\prime}=\left(\frac{3(\sin (2 k a)-2 k a \cos (2 k a))}{(2 k a)^{3}}\right)=F_{0}(k ; a)$,

and $F_{0}(k ; a)^{2}=F(k ; a)$ is the spherical form factor. ${ }^{13}$ From there, the scattering amplitude resulting from one aggregate is expressed as

$$
\Phi_{\mathrm{ag}}(k)=\frac{k^{2}\left(\gamma_{\kappa}-\gamma_{\rho}\right)}{4 \pi} V_{\mathrm{c}} F_{0}(k ; a) \sum_{j=1}^{N} e^{2 i k \mathbf{n}_{0} \cdot \mathbf{r}_{j}} .
$$




\section{The coherent component}

The coherent component of the differential backscattering cross-section of the effective scatterer is obtained from the coherent scattering amplitude as

$$
\sigma_{\mathrm{ag}, \mathrm{coh}}(k)=\left|\left\langle\Phi_{\mathrm{ag}}(k)\right\rangle\right|^{2},
$$

where $\langle\cdot\rangle$ represents the expected value of a random variable. One computes:

$$
\begin{aligned}
\left\langle\Phi_{\mathrm{ag}}(k)\right\rangle & =\frac{k^{2}\left(\gamma_{\kappa}-\gamma_{\rho}\right)}{4 \pi} V_{\mathrm{c}} F_{0}(k ; a) \sum_{j=1}^{N}\left\langle e^{2 i k \mathbf{n}_{0} \cdot \mathbf{r}_{j}}\right\rangle \\
& =\frac{k^{2}\left(\gamma_{\kappa}-\gamma_{\rho}\right)}{4 \pi} \phi_{i} V_{\mathrm{ag}} F_{0}(k ; a) \frac{1}{N} \sum_{j=1}^{N}\left\langle e^{\left.2 i k \mathbf{n}_{0} \cdot \mathbf{r}_{(} \phi\right)}\right.
\end{aligned}
$$

where $V_{\mathrm{ag}}=\frac{4}{3} \pi r_{\mathrm{ag}}^{3}$ is the volume of an aggregate of radius $r_{\mathrm{ag}}$, and $\phi_{i}=N V_{\mathrm{c}} / V_{\mathrm{ag}}$ is the aggregate compactness. Next, we define

$$
\begin{aligned}
& \rho_{\mathrm{ag}}=\phi_{i} \rho_{c}+\left(1-\phi_{i}\right) \rho_{0} \\
& \kappa_{\mathrm{ag}}=\left[\phi_{i} / \kappa_{c}+\left(1-\phi_{i}\right) / \kappa_{0}\right]^{-1}
\end{aligned}
$$

which are obtained from the average values of $1 / \rho$ and $\kappa$, respectively, within the aggregate, as mentioned in Eq. (8.2.23) of Morse \& Ingard. ${ }^{12}$ Denoting $\gamma_{\kappa, \text { ag }}=$ $\left(\kappa_{\text {ag }}-\kappa_{0}\right) / \kappa_{0}$ and $\gamma_{\rho, \text { ag }}=\left(\rho_{\text {ag }}-\rho_{0}\right) / \rho_{\text {ag }}$, one computes $\left(\gamma_{\kappa}-\gamma_{\rho}\right) \phi_{i}=\gamma_{\kappa, \text { ag }}-\gamma_{\rho, \text { ag }}$. Based on these definitions, it follows that the coherent scattering amplitude of the aggregate is expressed as:

$$
\left\langle\Phi_{\mathrm{ag}}(k)\right\rangle=\frac{k^{2}\left(\gamma_{\kappa, \mathrm{ag}}-\gamma_{\rho, \mathrm{ag}}\right)}{4 \pi} V_{\mathrm{ag}} F_{0}(k ; a) \frac{1}{N} \sum_{j=1}^{N}\left\langle e^{2 i k \mathbf{n}_{0} \cdot \mathbf{r}_{j}}\right\rangle .
$$

Note that in this derivation, no approximation was used, but rather algebraic manipulations and definitions.

The expression $\mathcal{F}_{\text {coh }}(k)=$ $F_{0}(k ; a) 1 / N \sum_{j=1}^{N}\left\langle e^{2 i k \mathbf{n}_{0} \cdot \mathbf{r}_{j}}\right\rangle$ corresponds to the Fourier transform of the function $\left\langle 1 / N \sum \chi_{a}\left(\mathbf{r}-\mathbf{r}_{j}\right)\right\rangle$ that describes a system of spheres, where $\chi_{a}\left(\mathbf{r}-\mathbf{r}_{j}\right)=1$ inside a sphere $\mathbf{V}_{a}$ of radius $a$ centered at $\mathbf{r}_{j}$, and 0 elsewhere. Since it is not straightforward to compute the expression $\mathcal{F}_{\text {coh }}(k)$, we approximate this quantity with the expression $F_{0}\left(k, r^{\prime}\right)$ given in Eq. (4) for an equivalent full sphere of radius $r^{\prime}$. The full sphere choice was motivated here by the postulated spherical distribution of cells in aggregates. In order to match the low frequency approximations of the scattering amplitudes from the system of spheres and from the equivalent full sphere, their gyration radii $R_{g}$ should be equal. The gyration radius of a system of $N$ spheres of radius $a$ is equal to

$$
R_{g}=\sqrt{\frac{3}{5} a^{2}+\frac{1}{N} \sum_{j=1}^{N}\left|\mathbf{r}_{j}\right|^{2}}
$$

where $\mathbf{r}_{j}$ is the position vector of the $j$ th cell with respect to the aggregate center, as given in Eq. (6) of Saha et $a l .{ }^{14}$ So, the radius $r^{\prime}$ of the equivalent full sphere must be expressed as: $r^{\prime}=\sqrt{\frac{5}{3}} R_{g}$, as demonstrated in Appendix B. Henceforth, from Eq. (8), the coherent scattering amplitude resulting from one aggregate is approximated as

$$
\left\langle\Phi_{\mathrm{ag}}(k)\right\rangle \approx \frac{k^{2}\left(\gamma_{\kappa, \mathrm{ag}}-\gamma_{\rho, \mathrm{ag}}\right)}{4 \pi} V_{\mathrm{ag}} F_{0}\left(k ; \sqrt{\frac{5}{3}} R_{g}\right) .
$$

All together, the coherent component of the differential backscattering cross-section of the effective sphere is modeled as:

$$
\sigma_{\mathrm{ag}, \mathrm{coh}}(k) \approx \frac{k^{4}\left(\gamma_{\kappa, \mathrm{ag}}-\gamma_{\rho, \mathrm{ag}}\right)^{2}}{16 \pi^{2}} V_{\mathrm{ag}}^{2} F\left(k ; \sqrt{\frac{5}{3}} R_{g}\right) .
$$

One should note that in the former work, ${ }^{10}$ the spherical form factor $F$ given in Eq. (11), was computed by considering the external radius of the aggregate $r_{\text {ag }}$ (instead of $\sqrt{5 / 3} R_{g}$ in the present work). Comparison between the former and the present coherent form factors will be presented in the subsection IV.A.

\section{The incoherent component}

Inside the aggregate, each cell produces a scattered wave as a sphere of radius $a$, with density $\rho_{c}$ and compressibility $\kappa_{c}$, in a surrounding medium of density $\rho_{\text {ag }}$ and compressibility $\kappa_{\text {ag }}$ throughout the aggregate. Therefore, the wavenumber inside the aggregate is modified as $^{12} k_{\text {ag }}=k \sqrt{\rho_{\text {ag }} \kappa_{\text {ag }}} / \sqrt{\rho_{0} \kappa_{0}}$. Based on Eq. (5), the incoherent component of the differential backscattering cross-section of the effective scatterer is equal to

$$
\begin{aligned}
\sigma_{\mathrm{ag}, \text { inc }}(k) & =\left\langle\left|\Phi_{\mathrm{ag}}\left(k_{\mathrm{ag}}\right)-\left\langle\Phi_{\mathrm{ag}}\left(k_{\mathrm{ag}}\right)\right\rangle\right|^{2}\right\rangle \\
& =\frac{k_{\mathrm{ag}}^{4}\left(\gamma_{\kappa}-\gamma_{\rho}\right)^{2}}{16 \pi^{2}} V_{\mathrm{c}}^{2} N F\left(k_{\mathrm{ag}} ; a\right) S_{0}\left(k_{\mathrm{ag}} ; a, \phi 1 \beta,\right)
\end{aligned}
$$

where

$$
S_{0}\left(k ; a, \phi_{i}\right)=\left\langle\frac{1}{N}\left|\sum_{j=1}^{N} e^{2 i k \mathbf{n}_{0} \cdot \mathbf{r}_{j}}-\sum_{j=1}^{N}\left\langle e^{2 i k \mathbf{n}_{0} \cdot \mathbf{r}_{j}}\right\rangle\right|^{2}\right\rangle
$$

is the structure factor for a collection of $N$ randomly distributed identical spheres of radius $a$ and of concentration $\phi_{i}$ within an aggregate. Assuming that the aggregate is sufficiently large, Eq. (14) corresponds to Eq. (10) in Ref. 15. This latter structure factor was analytically calculated as established by Wertheim, ${ }^{16}$ which is denoted here $S\left(k_{\mathrm{ag}} ; a, \phi_{i}\right)$. One thus obtains the expression

$$
\sigma_{\mathrm{ag}, \text { inc }}(k) \approx \frac{k_{\mathrm{ag}}^{4}\left(\gamma_{\kappa}-\gamma_{\rho}\right)^{2}}{16 \pi^{2}} V_{\mathrm{ag}} V_{\mathrm{c}} \phi_{i} F\left(k_{\mathrm{ag}} ; a\right) S\left(k_{\mathrm{ag}} ; a, \phi_{i}\right) .
$$

In the sequel, we compute the differential backscattering cross-section $\sigma_{\mathrm{ag}}=\sigma_{\mathrm{ag}, \mathrm{coh}}+\sigma_{\mathrm{ag}, \mathrm{inc}}$ of an aggregate using 
Eqs. (1), (11) and (15).

Let us mention that one can derive an alternative expression of the incoherent component of the differential backscattering cross-section based on a Gaussian direct correlation function $\sigma_{\mathrm{ag}, \text { inc, } \mathrm{G}}$ as proposed by Morse and Ingard. ${ }^{12}$ The proposed computation of $\sigma_{\text {ag,inc, } \mathrm{G}}$ is a modified version of the incoherent component of Ref. 12 and is expressed as follows:

$\sigma_{\mathrm{ag}, \mathrm{inc}, \mathrm{G}}(k) \approx \frac{k_{\mathrm{ag}}^{4}\left(\gamma_{\kappa}-\gamma_{\rho}\right)^{2}}{16 \pi^{2}} V_{\mathrm{ag}} \phi_{i}\left(1-\phi_{i}\right) \frac{8 \sqrt{2} \pi^{3 / 2}}{3} d^{5} k_{\mathrm{ag}}^{2} e^{-d^{2} k_{\mathrm{a}}}$

where $d$ is the correlation distance related to the cell radius $a$ as $d=\frac{2^{1 / 6}}{3^{1 / 3} \pi^{1 / 6}} a \approx 0.643092 \times a$, as proposed by Insana and Brown (see Eq. (81) p.107 in Ref. 13). Details can be found in Appendix C. The differential backscattering cross-section of an aggregate based on Eqs. (1), (11) and (16) is denoted $\sigma_{\mathrm{ag}, \mathrm{G}}$ in the sequel.

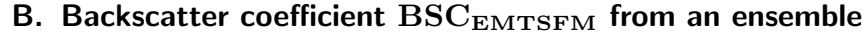 of identical aggregates}

The BSC from an ensemble of identical aggregates is obtained by summing the contributions from individual effective scatterers and modeling the effective sphere interaction by a statistical mechanics structure factor $S_{\mathrm{ag}}$ as follows: ${ }^{10,11}$

$$
\operatorname{BSC}_{\text {EMtSFM }}(k)=m_{\mathrm{ag}} \sigma_{\mathrm{ag}}(k) S_{\mathrm{ag}}\left(k ; r_{\mathrm{ag}}, \phi_{\mathrm{ag}}\right),
$$

where $m_{\mathrm{ag}}$ is the number density of effective spheres, which is related to the volume fraction of effective spheres $\phi_{\mathrm{ag}}$ as $m_{\mathrm{ag}}=\phi_{\mathrm{ag}} / V_{\mathrm{ag}}$ and $\sigma_{\mathrm{ag}}$ is computed using Eqs. (1), (11) and (15). In Eq. (17), $S_{\text {ag }}$ is the structure factor for a collection of randomly distributed identical effective spheres of radius $r_{\mathrm{ag}}$ and of concentration $\phi_{\mathrm{ag}}$, analytically computed as established by Wertheim. ${ }^{16}$ The volume fraction of effective scatterers is equal to the volume fraction of cells in the tissue $\phi$ divided by the aggregate compactness $\phi_{i}: \phi_{\mathrm{ag}}=\phi / \phi_{i}$.

\section{3D SIMULATION METHOD}

The 3D simulation study was conducted based on the Structure Factor Model (SFM). The SFM is an ultrasound scattering model largely used to predict the frequency dependence of the BSC from aggregated cells in the field of ultrasound tissue characterization. . $^{4,8,10,14,17}$ The SFM consists of summing contributions from individual cells and modeling cell interaction by a statistical mechanics structure factor, whatever the complexity of the cells spatial distribution such as cell aggregates. The structure factor computation for a complex particle spatial distribution was described in details in Section 6.3.1 of Ref. 17. The current section focuses on the procedure for obtaining cell aggregates in the computer simulations, and then on the simulation of the differential backscattering cross section of one cell aggregate, denoted $\sigma_{\mathrm{ag}, \mathrm{sim}}$, and of the $\mathrm{BSC}_{\text {sim }}$ from aggregated cells based on the SFM.

\section{A. Simulation of spatial distribution of cells within one aggregate}

We describe here how the cell distribution inside one $2^{2} k_{\text {agg }}$ agregate was computed. The cell particle radius $a$ was set to $2.75 \mu \mathrm{m}$ for all simulations, which corresponds to the red blood cell size usually used in blood computer simulations. ${ }^{11}$ We specified the aggregate radius $r_{\mathrm{ag}}$ defined as the radius of the external envelope (Fig. 1) and the aggregate compactness $\phi_{i}$ that fixes the number of cells $N$ within the aggregate. $N$ cells were uniformly randomly distributed such that cells within a radius of $r_{\mathrm{ag}}$ could overlap, and the total number of overlapping pairs was counted. Then, the system was able to evolve by moving randomly selected cells to another position than the initial. If the number of overlapping pairs of the new system equaled or was lower than in the previous system, the displacement was accepted and the procedure was repeated until no overlapping was detected. This process allowed to reach aggregate compactness $\phi_{i}$ up to $40 \%$ in $3 \mathrm{D}$, whereas a method using a random sequential absorption $^{18}$ would give compactness near $30 \%$. Figure 1 illustrates spatial arrangements of cells within a single aggregate for two aggregate compactnesses of $10 \%$ and $40 \%$ with an identical aggregate radius $r_{a g} / a=7$. The radius of gyration was computed using Eq. (9). For the aggregate example given in Fig. 1, a change of compactness from $10 \%$ to $40 \%$ induces a slight increase of the radius of gyration from $R_{\mathrm{g}}=12.56 \mu \mathrm{m}$ to $R_{\mathrm{g}}=14.02$ $\mu \mathrm{m}$.

\section{B. Computation of a simulated $\sigma_{\mathrm{ag}, \operatorname{sim}}$ curve}

The simulated differential backscattering cross section of one cell aggregate $\sigma_{\mathrm{ag}, \mathrm{sim}}$, was computed using the $\mathrm{SFM}^{10,11}$ as follows

$$
\sigma_{\mathrm{ag}, \operatorname{sim}}(k)=N \sigma_{b}(k) S_{\mathrm{sim}}^{\prime}(k),
$$

where $\sigma_{b}$ is the differential backscattering cross section of a single cell given by the fluid-filled sphere expression ${ }^{12,13}$

$$
\sigma_{b}(k)=\frac{k^{4} V_{\mathrm{c}}^{2} \gamma_{z}^{2}}{4 \pi^{2}} F(k ; a),
$$

and $S_{\text {sim }}^{\prime}$ is the simulated structure factor representing the spatial positioning of the cells inside the aggregate and defined as

$$
S_{\text {sim }}^{\prime}(k)=\left\langle\frac{1}{N}\left|\sum_{j=1}^{N} e^{-2 i k \mathbf{n}_{0} \cdot \mathbf{r}_{j}}\right|^{2}\right\rangle
$$


where the cell positions $\mathbf{r}_{j}$ are given using the procedure described in section III.A. The simulated structure factor $S_{\mathrm{sim}}^{\prime}$ was determined from the 3D Fourier transform of the spatial distribution of cells. ${ }^{17} \mathrm{~A}$ mean $\sigma_{\mathrm{ag}, \mathrm{sim}}$ curve was computed by averaging over 1000 different realizations for averaging purposes.

\section{Computation of a simulated $B S C$ from several aggregates}

Random distributions of non-overlapping aggregates were computed within the simulated cubical volume $480^{3}$ $\mu \mathrm{m}^{3}$ by specifying the volume fraction $\phi$ of cells of radius $a$, the aggregate radius $r_{\mathrm{ag}}$ and the aggregate compactness $\phi_{i}$. The spatial distribution of cells inside each aggregate was performed using the procedure described in section III.A. The simulated cubical volume was periodized; i.e., interactions between aggregates were determined under periodic boundary conditions, in order to remove the edge effects. The BSC from aggregated cells was then computed using the SFM as follows

$$
\mathrm{BSC}_{\operatorname{sim}}(k)=\frac{\phi}{V_{\mathrm{c}}} \sigma_{b}(k) S_{\mathrm{sim}}(k),
$$

where $S_{\text {sim }}$ is the simulated structure factor representing the spatial positioning of the cells inside the simulated tissue. For each distribution of cells, the 3-D Fourier Transform of the cell spatial positioning was computed to obtain the simulated structure factor. ${ }^{17}$. The mean $\mathrm{BSC}_{\text {sim }}$ curve was obtained by averaging over 100 realizations for averaging purposes. Only 100 realizations were used here (against 1000 realizations in the case of the $\sigma_{\text {ag,sim }}$ computation) because one simulated volume involve several thousands of cells (against a few dozen of cells per aggregate for the $\sigma_{\mathrm{ag}, \mathrm{sim}}$ computation).

\section{RESULTS}

\section{A. Comparison of the simulated and theoretical $\sigma_{\text {ag }}$}

The frequency-dependent differential backscattering cross section of one cell aggregate $\sigma_{\mathrm{ag}, \mathrm{sim}}$ computed with the SFM is given in Fig. 2 for a single aggregate of radius $r_{\mathrm{ag}} / a=6$ and of compactness $\phi_{i}=30 \%$. Also shown in Fig. 2(a) (and Fig. 2(b), respectively) are the theoretical differential backscattering cross section considering only the coherent component (and considering both coherent and incoherent components, respectively). In Fig. 2(a), the dashed line (or the solid line, respectively) represents the theoretical $\sigma_{\text {ag,coh }}$ given in Eq. (11) using $F\left(r_{\text {ag }}\right)$ (or $F\left(\sqrt{5 / 3} R_{\mathrm{g}}\right)$, respectively). The first peaks of $\sigma_{\mathrm{ag}, \mathrm{sim}}$ and

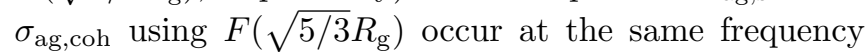
(approximately $22.1 \mathrm{MHz}$ ). On the other hand, the first peak of $\sigma_{\text {ag,coh }}$ using $F\left(r_{\mathrm{ag}}\right)$ does not match perfectly the simulation and occurs at a lower frequency around 20.5 $\mathrm{MHz}$.
In Fig. 2(b), the solid line represents $\sigma_{\mathrm{ag}}=$ $\sigma_{\mathrm{ag}, \mathrm{coh}}+\sigma_{\mathrm{ag}, \text { inc }}$ using Eqs. (1), (11) and (15) and the dashed line represents $\sigma_{\mathrm{ag}, \mathrm{G}}=\sigma_{\mathrm{ag}, \mathrm{coh}}+\sigma_{\mathrm{ag}, \text { inc }, \mathrm{G}}$ using Eqs. (1), (11) and (16). The consideration of the incoherent component allows to better match the $\sigma_{\mathrm{ag}, \mathrm{sim}}$ behavior at higher frequencies, whatever the considered expression for the incoherent component $\sigma_{\mathrm{ag}, \text { inc }}$ or $\sigma_{\mathrm{ag}, \mathrm{inc}, \mathrm{G}}$. Indeed, the $\sigma_{\mathrm{ag}, \mathrm{coh}}$ curve shows very deep dips (Fig. 2(a)), whereas both $\sigma_{\text {ag }}$ and $\sigma_{\text {ag,G }}$ curves match much better the dip behavior of the $\sigma_{\mathrm{ag}, \mathrm{sim}}$ curve.

Figure 3 represents the simulated $\sigma_{\text {ag,sim }}$ (symbols) for a radius $r_{\mathrm{ag}} / a=6$ and aggregate compactnesses $\phi_{i}=10,20,30$ and $40 \%$. One can note that the $\sigma_{\mathrm{ag}, \mathrm{sim}}$ curves have more pronounced frequency dips when the aggregate compactness increases. For the lower aggregate compactness, only the first peak is well pronounced and the first dip is smooth, whereas the first two peaks and the first dip are clearly enhanced for the higher aggregate compactnesses. This behavior suggests that for compact aggregates, the incident wave tends to be scattered as if the aggregate was a well defined sphere, whereas for the lower aggregate compactness, the boundaries of the aggregates are not well defined. The occurence of the first frequency peak also shifts toward higher frequencies from $22.6 \mathrm{MHz}$ to $25.8 \mathrm{MHz}$ when the aggregate compactness decreases from $40 \%$ to $10 \%$. Also represented in Fig. 3 are the theoretical $\sigma_{\text {ag }}$ (solid line) and $\sigma_{\mathrm{ag}, G}$ (dashed line). Both models are very accurate at $\phi_{i}=30 \%$. The $\sigma_{\mathrm{ag}, \mathrm{G}}$ curve matches better the simulation results for the highest aggregate compactness $\phi_{i}=40 \%$, whereas the $\sigma_{\text {ag }}$ curve matches better the two lower compactnesses $\phi_{i}=10 \%$ and $20 \%$.

Figure 3 also allows to compare the general $\sigma_{\text {ag }}$ behavior over all frequencies, however, in order to evaluate a workable frequency bandwidth for each model, the product $k r_{a g}$ for which the relative error was less than $20 \%$ was determined, as done previously in our twodimensional study (see Fig. 7 of Franceschini et al. ${ }^{10}$ ). First, we assess the improvement of the $k r_{a g}$ limit for the $\sigma_{\text {ag,coh }}$ expression using $F\left(\sqrt{5 / 3} R_{\mathrm{g}}\right)$ (see the solid curves in Fig. (4)). In comparison with the former modeling using $F\left(r_{\mathrm{ag}}\right)$, the use of $F\left(\sqrt{5 / 3} R_{\mathrm{g}}\right)$ increases the $k r_{a g}$ limit from 1.07 to 1.76 on average for $\phi_{i}=10 \%$, and from 1.47 to 1.91 on average for $\phi_{i}=40 \%$ (the limit being computed as the mean $k r_{a g}$ for $r_{\text {ag }} / a$ values varying from 5 to 9 ). Secondly, we focus on the improvement of the $k r_{a g}$ limit considering the incoherent component expressions (see the dashed curves in Fig. (4)). For the lowest aggregate compactness of $10 \%$, consideration of the incoherent component based on the structure factor $\sigma_{\text {ag,inc }}$ allowed to increase the $k r_{a g}$ limit from 1.76 to 2.12 , whereas the incoherent component based on the Gaussian direct correlation function $\sigma_{\text {ag,inc, },}$ did not allow to improve the $k r_{a g}$ limit. For the highest aggregate compactness of $40 \%$, there was no significant difference 
in the $k r_{a g}$ limit whether the incoherent component was considered or not: it means that the $k r_{a g}$ limit increase is only due to the modification of $F\left(\sqrt{5 / 3} R_{\mathrm{g}}\right)$ in the coherent component. To conclude, the overall best results are obtained by taking into account (1) the modification of $F\left(\sqrt{5 / 3} R_{\mathrm{g}}\right)$ in the coherent component, and (2) the incoherent component based on the structure factor $\sigma_{\text {ag,inc. }}$. Thus, in the sequel, for the results presented later, we will use the differential backscattering cross section $\sigma_{\text {ag }}$ computed with Eqs. (1), (11) and (15).

\section{B. Comparison of the simulated and theoretical BSC}

Figure 5 (a) shows simulated and theoretical frequency dependent BSCs for three aggregate sizes $r_{\mathrm{ag}} / a=5,7$ and 9 , a fixed aggregate compactness $\phi_{i}=40 \%$ and a fixed cell volume fraction $\phi=16 \%$. The symbols represent the $\mathrm{BSC}_{\text {sim }}$ computed with the SFM. The solid and dashed lines depicts the $\mathrm{BSC}_{\text {EMTSFM }}$ using the proposed model of Eqs. (1), (11), (15), and (17). The theoretical

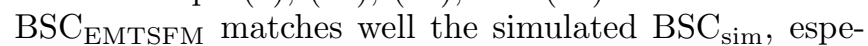
cially for the amplitude and the frequency occurence of the first two peaks and dips, whatever the aggregate size considered in this work. In Fig. 5(b) are shown simulated and theoretical BSCs for three aggregate compactnesses $\phi_{i}=10,25$ and $40 \%$, at a lower $\phi=4 \%$, and a fixed aggregate radius of $r_{\mathrm{ag}} / a=7$. Note that for the study of the aggregate compactness variation, we limit the total cell volume fraction to $\phi=4 \%$ and the lower aggregate compactness to $\phi_{i}=10 \%$ (corresponding to a value $\phi_{\text {ag }}=40 \%$, because the maximum volume fraction of aggregates achievable in computer simulation is approximately $40 \%$ using the method described section III.C.

The influence of the structure factor of the effective medium $S_{\text {ag }}$ on the BSC curves was also studied. In that aim, we observe the spectral slope (i.e. the linear slope of the BSC as a function of frequency in a log$\log$ scale) of the $\mathrm{BSC}_{\text {sim }}$ curves before the first peak occurrence. The spectral slope increases above 4 for the $\mathrm{BSC}_{\text {sim }}$ curves with an aggregate concentration $\phi_{\mathrm{ag}}=$ $\phi / \phi_{i}=40 \%$ presented in Fig. 5(a), and for the $\mathrm{BSC}_{\mathrm{sim}}$ curve with the clustering condition $\left(\phi=4 \%, \phi_{i}=10 \%\right.$, $\left.\phi_{\mathrm{ag}}=\phi / \phi_{i}=40 \%\right)$ presented in Fig. 5(b). On the other hand the $\mathrm{BSC}_{\text {sim }}$ curve shows a fourth power frequencydependence (i.e. a spectral slope of 4 ) with the clustering condition $\left(\phi=4 \%, \phi_{i}=40 \%, \phi_{\mathrm{ag}}=\phi / \phi_{i}=10 \%\right)$ presented in Fig. 5(b). So, the more the aggregate concentration $\phi_{\text {ag }}$ increases, the greater the influence of $S_{\text {ag }}$ becomes observable in the BSC frequency dependence.

\section{DISCUSSION}

\section{A. On the use of simple spatial distribution of cells}

The method used in this study to obtain the cell spatial distribution was not based on a generic physical model of interactions between cells. Rather, we used a simple and fast method to generate hundreds of simulated media with a large scale ratio between the cell size $(2.75 \mu \mathrm{m})$ and the whole medium size $\left(480^{3} \mu \mathrm{m}^{3}\right)$. The purpose was to build a controlled medium containing non-overlapping spherical aggregates, all aggregates having the same radius and compactness with a unique cell spatial distribution defined by a structure factor. The main advantage of this method was the possibility to have various aggregate compactnesses with the same size of aggregates, which allowed to demonstrate the role of the incoherent scattering component on the BSC frequency dependence at high frequencies. Indeed, the $\sigma_{\text {ag,sim }}$ curves had well pronounced peaks and dips for the higher aggregate compactnesses, whereas the first peak was well enhanced and the first dip was smooth for the lower aggregate compactness (see Fig. 3). Theoretical predictions of the $\sigma_{\text {ag }}$ based on the structure factor for the incoherent component proposed in Eq. (15) agreed well with the simulations and validated the proposed theoretical modeling.

In the 3-D computer simulations of this study, we had to limit the total volume fraction of cells to a maximum of $16 \%$. Indeed, the procedure we chose to distribute the cells within aggregates allowed reaching a maximum aggregate compactness $\phi_{i, \max }$ of $40 \%$. The upper limit of $40 \%$ is easily understandable because placing nondeformable identical spheres randomly enough inside a larger sphere without overlapping is not straightforward. In comparison, a standard method using a random sequential absorption ${ }^{18}$ would give a compactness close to $30 \%$. The maximum aggregate volume fraction $\phi_{\mathrm{ag}, \max }$ was thus also fixed to $40 \%$. As a consequence, the maximum value of the total volume fraction $\phi_{\max }$ was limited to $\phi_{\max }=\phi_{i, \max } \phi_{\mathrm{ag}, \max }=(40 \%)^{2}=16 \%$.

\section{B. The benefit of considering the incoherent component}

A new theoretical modeling of the differential backscattering cross section from a single cell aggregate was developed in this work and consisted in taking into account a coherent component and an incoherent component in the expression of $\sigma_{\text {ag }}$. This modeling was compared to numerical simulations based on the SFM. Concerning the coherent component, a slight modification of the $\sigma_{\text {ag,coh }}$ expression using $F\left(\sqrt{5 / 3} R_{\mathrm{g}}\right)$ was proposed and allowed the EMT to better match the frequency occurrences of peaks and dips observed in simulations. Concerning the incoherent component, two expressions of $\sigma_{\text {ag,inc }}$ based on the structure factor and on a Gaussian direct correlation function proposed by Morse and Ingard $^{12}$ were compared. The numerical 
study demonstrated the superiority of the formulation using the structure factor over that with the Gaussian direct correlation function, regarding the $k r_{a g}$ limit study of Fig. 4. The consideration of the incoherent component $\sigma_{\text {ag,inc }}$ based on the structure factor allowed to approximate the simulations satisfactorily for an average $k r_{a g}$ limit around 2, against an average $k r_{a g}$ limit comprised between 1.07 and 1.47 with the former model considering only the coherent component.

It is interesting to observe that the simulated $\sigma_{\mathrm{ag}, \mathrm{sim}}$ curves showed a fourth power frequency dependence (Rayleigh scattering) before the first peak occurrence (see Figures 2 and 3). When the aggregate compactness varied (Fig. 3), the $\sigma_{\text {ag,sim }}$ frequency dependence differed mainly at high frequencies after the first peak. To better understand this high frequency behavior, we analyzed the theoretical respective influence of each scattering component (coherent and incoherent) on the $\sigma_{\text {ag }}$ behavior, while varying the aggregate compactness. According to Eqs. (11) and (15), the $\sigma_{\text {ag }}$ frequency dependence is mainly determined by the spherical form factor from an effective sphere $F\left(\sqrt{5 / 3} R_{\mathrm{g}}\right)$ that intervenes in the coherent component, and the structure factor $S\left(a, \phi_{i}\right)$ that intervenes in the incoherent component. As shown in Fig. 6(a), an increase of the compactness induces a decrease of the structure factor amplitude $S\left(k ; a, \phi_{i}\right)$, and a slight shift toward lower frequencies of the form factor $F\left(k ; r_{\text {ag }}^{\prime}\right)$ peaks and dips. In Fig. 6(b) (or (c), respectively) are plotted the theoretical backscattering cross sections (solid lines), the corresponding coherent component $\sigma_{\text {ag,coh }}$ (dashed lines) and incoherent component $\sigma_{\text {ag,inc }}$ (dotted lines) as defined in Eq. (1), for the same aggregating condition with $r_{\text {ag }} / a=6$ and $\phi_{i}=10 \%$ (or $\phi_{i}=40 \%$, respectively). The relative influence of the incoherent component is more important for lower aggregate compactnesses, giving rise to smoother peaks and dips. Also, the frequency shift of the peaks and dips observed in the simulations can be explained theoretically by a dependance of the form factor $F\left(\sqrt{5 / 3} R_{\mathrm{g}}\right)$ on the aggregate compactness. Indeed, the gyration radius value depends on the aggregate compactness as mentioned in section III.A. In the framework of an inverse problem approach, it would be useful to approximate the gyration radius as a function of the radius $r_{\mathrm{ag}}$ of the aggregate envelope and the aggregate compactness $\phi_{i}$. Based on the 3D building of isotropic aggregates using the method described in section III.A, the relationship between $R_{g}$, $r_{\text {ag }}$ and $\phi_{i}$ was empirically determined as follows:

$$
R_{\mathrm{g}} \approx \sqrt{3 / 5}\left(0.96 \times r_{\mathrm{ag}}+7.06 \times 10^{-6} \phi_{i}-2.71 \times 10^{-6}\right)
$$

This approximation, obtained from a linear regression analysis, shows less than $5 \%$ relative error for all tested radii $r_{\text {ag }} / a$ (varying from 4 to 9) and compactnesses (varying from $5 \%$ to $40 \%$ ). The estimated R-squared value and $\mathrm{p}$-value were 0.996 and $5.39 \times 10^{-101}$ respec- tively.

\section{CONCLUSION}

The EMT was further developed to decompose the differential backscattering cross section of a single cell aggregate into coherent and incoherent components. The coherent component, corresponding to the average wave emerging from the effective scatterer, was approximated by the scattering of an effective fluid sphere, whose radius depends on the gyration radius of the cell aggregate. An important contribution of this new EMT is the taking into account of the incoherent component based on the structure factor, which allowed to approximate the computer simulations satisfactorily for a product $k r_{\text {ag }}$ up to 2 .

The EMTSFM assumes that all cells are aggregated and that aggregates are identical and isotropic. Therefore, the BSC behavior obtained in all simulations showed a pronounced first peak. In experimental conditions when insonifying aggregated red blood cells ${ }^{9}$ or clusters of tumor cells ${ }^{5}$, the BSC behavior was smoother and the peaks were less pronounced. The reason behind this might be that real tissues contain several sizes of aggregates, and since the location of BSC peaks are different for different aggregate populations, a relatively smoother BSC curve can be obtained. Therefore, future improvements should consider incorporating polydispersity in aggregate size and compactness to provide an optimal EMTSFM for the inversion of experimental data.

\section{ACKNOWLEDGMENT}

This research was supported by the Canadian Institutes of Health Research (grant \#MOP-84358), the CNRS, the CSIR New Delhi, as well as the Labex MEC (ANR-10-LABX-0092) and the A*MIDEX project (ANR-11-IDEX-0001-02) funded by the Investissements d'Avenir French Government program managed by the French National Research Agency (ANR). F. Destrempes was an invited researcher at the Laboratoire de Mécanique et d'Acoustique LMA, CNRS, UPR 7051, Aix-Marseille University, Centrale Marseille, F-13402 Marseille Cedex 20, France, in July 2013 to work on this project.

\section{Appendix A}

The model underlying Eq. (2) is equivalent to the following expression for the tissue function

$$
\gamma_{\kappa}\left(\mathbf{r}_{0}\right)-\gamma_{\rho}\left(\mathbf{r}_{0}\right)=\left(\gamma_{\kappa}-\gamma_{\rho}\right) \sum_{j=1}^{N} \chi\left(\mathbf{r}_{0}-\mathbf{r}_{j}\right)
$$

where $\chi\left(\mathbf{r}_{0}\right)=1$ inside a sphere $\mathbf{V}_{a}$ of radius $a$ centered at the origin, and 0 elsewhere. 
We then compute based on Eq. (23)

Firstly, one has

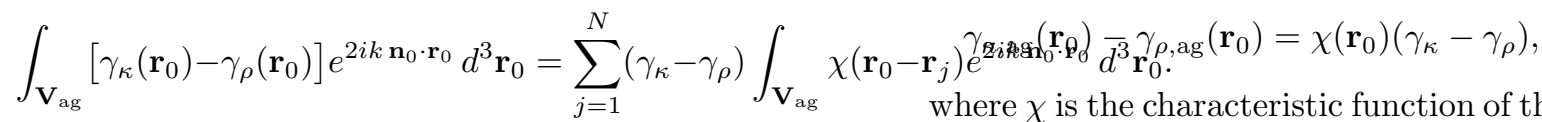

Next, considering the change of variable $\mathbf{r}_{0}^{\prime}=\mathbf{r}_{0}-\mathbf{r}_{j}$, one obtains

$$
\int_{\mathbf{V}_{\text {ag }}} \chi\left(\mathbf{r}_{0}-\mathbf{r}_{j}\right) e^{2 i k \mathbf{n}_{0} \cdot \mathbf{r}_{0}} d^{3} \mathbf{r}_{0}=\int_{\mathbf{V}_{a}} e^{2 i k \mathbf{n}_{0} \cdot\left(\mathbf{r}_{0}^{\prime}+\mathbf{r}_{j}\right)} d^{3} \mathbf{r}_{0}^{\prime} .
$$
1 inside cells and $\chi\left(\mathbf{r}_{0}\right)=0$, otherwise. Moreover, we use the following approximation

$$
\left\langle\gamma_{\kappa, \mathrm{ag}}\left(\mathbf{r}_{0}\right)-\gamma_{\rho, \mathrm{ag}}\left(\mathbf{r}_{0}\right)\right\rangle \approx \phi_{i}\left(\gamma_{\kappa}-\gamma_{\rho}\right) .
$$

Lastly, since $e^{2 i k \mathbf{n}_{0} \cdot\left(\mathbf{r}_{0}^{\prime}+\mathbf{r}_{j}\right)}=e^{2 i k \mathbf{n}_{0} \cdot \mathbf{r}_{0}^{\prime}} e^{2 i k \mathbf{n}_{0} \cdot \mathbf{r}_{j}}$, one concludes that

From there, one obtains the expression

$$
\int_{\mathbf{V}_{\mathrm{ag}}}\left[\gamma_{\kappa}\left(\mathbf{r}_{0}\right)-\gamma_{\rho}\left(\mathbf{r}_{0}\right)\right] e^{2 i k \mathbf{n}_{0} \cdot \mathbf{r}_{0}} d^{3} \mathbf{r}_{0}=\left(\gamma_{\kappa}-\gamma_{\rho}\right)\left(\int_{\mathbf{V}_{a}} e^{2 i k \mathbf{n}_{0} \cdot \mathbf{r}_{0}^{\prime}} d^{3} \mathbf{r}_{0}^{\prime}\right) \sum_{j=1}^{N} \underset{4 \pi}{\stackrel{k_{\mathrm{ag}}^{2}}{2 i \mathbf{m}_{0}} \cdot f_{V_{\mathrm{ag}}}^{2}}\left(\gamma_{\kappa}-\gamma_{\rho}\right)\left(\chi\left(\mathbf{r}_{0}\right)-\phi_{i}\right) e^{2 i k_{\mathrm{ag}} \mathbf{n}_{0} \cdot \mathbf{r}_{0}} d^{3} \mathbf{r}_{0}
$$

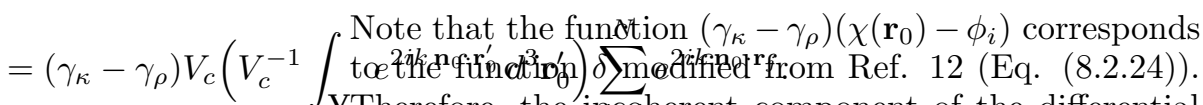

$\mathrm{V}_{\mathrm{T}}$ Therefore, the ingeaherent component of the differential backscattering cross-section can be computed using the

\section{Appendix B}

This appendix gives the computation steps to obtain the low frequency approximation of $\mathcal{F}_{\text {coh }}(k)=$ $F_{0}(k ; a) 1 / N \sum_{j=1}^{N}\left\langle e^{2 i k \mathbf{n}_{0} \cdot \mathbf{r}_{j}}\right\rangle$.

Firstly, by assuming centrosymetric aggregates, one has

$$
\begin{aligned}
\frac{1}{N} \sum_{j=1}^{N}\left\langle e^{2 i k \mathbf{n}_{0} \cdot \mathbf{r}_{j}}\right\rangle & =\frac{1}{N} \sum_{j=1}^{N}\left\langle\cos \left(2 k \mathbf{n}_{0} \cdot \mathbf{r}_{j}\right)\right\rangle \\
& =\frac{1}{N} \sum_{j=1}^{N}\left\langle\frac{\sin \left(2 k\left|\mathbf{r}_{j}\right|\right)}{2 k\left|\mathbf{r}_{j}\right|}\right\rangle \\
& \approx 1-\frac{2}{3} k^{2}\left\langle\frac{1}{N} \sum_{j=1}^{N}\left|\mathbf{r}_{j}\right|^{2}\right\rangle,
\end{aligned}
$$

where the last approximation corresponds to the second order Taylor expansion of the expression. An equivalent approach can be found in Guinier \& Fournet ${ }^{19}$ pp. 7 and 8. A second order Taylor expansion of $\mathcal{F}_{\text {coh }}(k)$ thus gives:

$$
\mathcal{F}_{\text {coh }}(k) \approx 1-\frac{2}{3} k^{2}\left(\frac{3}{5} a^{2}+\left\langle\frac{1}{N} \sum_{j=1}^{N}\left|\mathbf{r}_{j}\right|^{2}\right\rangle\right)=1-\frac{2}{3} k^{2} R_{g}^{2}
$$

by using the definition of $R_{g}$ given in Eq. (9), and the Taylor expansion of $F_{0}(k ; a)\left(\approx 1-\frac{2}{5} k^{2} a^{2}\right)$. Lastly, since for the equivalent full sphere of radius $r^{\prime}, F_{0}\left(k ; r^{\prime}\right) \approx$ $1-\frac{2}{5} k^{2} r^{\prime 2}$, we need $r^{\prime}=\sqrt{(5 / 3)} R_{g}$ in order to match the low frequency approximations of the form factors $\mathcal{F}_{\text {coh }}(k)$ and $F_{0}\left(k ; r^{\prime}\right)$.

\section{Appendix C}

This appendix gives the computation steps to obtain the incoherent component of the differential backscattering cross-section $\sigma_{\mathrm{ag}, \text { inc, G }}$ based on a Gaussian direct correlation function as proposed by Morse and Ingard. ${ }^{12}$ direct correlation function $\Upsilon$ of the compressibility and density fluctuations as follows:

$$
\begin{aligned}
\sigma_{\text {ag,inc }}(k) & =\left\langle\left|\Phi_{\text {ag,inc }}(k)\right|^{2}\right\rangle \\
& \approx \frac{k_{\mathrm{ag}}^{4}\left(\gamma_{\kappa}-\gamma_{\rho}\right)^{2}}{16 \pi^{2}} V_{\mathrm{ag}} \int_{\mathbf{V}_{\mathrm{ag}}} \Upsilon(\boldsymbol{\Delta} \mathbf{r}) e^{2 i k_{\mathrm{ag}} \mathbf{n}_{0} \cdot \boldsymbol{\Delta} \mathbf{r}} d^{3}(\mathbf{\Delta})
\end{aligned}
$$

where

$$
\Upsilon(\boldsymbol{\Delta} \mathbf{r})=\frac{1}{V_{\mathrm{ag}}}\left\langle\int_{\mathbf{V}_{\mathrm{ag}}}\left(\chi\left(\mathbf{r}_{0}\right)-\phi_{i}\right)\left(\chi\left(\mathbf{r}_{0}+\boldsymbol{\Delta} \mathbf{r}\right)-\phi_{i}\right) d^{3} \mathbf{r}_{0}\right\rangle .
$$

The function $\Upsilon$ depends only on $\Delta r=|\Delta \mathbf{r}|$ and must have the following properties: the mean value of $\Upsilon(\boldsymbol{\Delta r})$ is zero and $\lim _{|\boldsymbol{\Delta} \mathbf{r}| \rightarrow \infty} \Upsilon(\boldsymbol{\Delta} \mathbf{r})=0$. Under the postulate of a Gaussian model, the expression of the direct correlation function $\Upsilon$ is given by Morse \& Ingard $^{12}$ (Eq. (8.2.26)):

$$
\Upsilon(\Delta r) \approx \Upsilon(0)\left(1-\frac{\Delta r^{2}}{3 d^{2}}\right) e^{-\Delta r^{2} /\left(2 d^{2}\right)},
$$

where $\Upsilon(0)=\phi_{i}\left(1-\phi_{i}\right)$, and the correlation distance $d$ is related to the cell radius $a$ as $d=\frac{2^{1 / 6}}{3^{1 / 3} \pi^{1 / 6}} a \approx 0.643092 \times$ $R_{g}^{2} a$ as defined by Insana and Brown ${ }^{13}$ (Eq. (81) p.107). Gaussian incoherent component expressed in Eq. (16).

1 F. L. Lizzi, M. Astor, T. Liu, C. Deng, D. J. Coleman, and R. H. Silverman, "Ultrasonic spectrum analysis for tissue assays and therapy evaluation", Int. J. Imaging Syst. Technol. 8, 3-10 (1997).

2 M. F. Insana, R. F. Wagner, D. G. Brown, and T. J. Hall, "Describing small-scale structure in random media using pulse-echo ultrasound", J. Acoust. Soc. Am. 87 179-192 (1990).

3 R. M. Vlad, R. K. Saha, N. M. Alajez, S. Ranieri, G. J. Czarnota, and M. C. Kolios, "An increase in cellular size variance contributes to the increase in ultrasound backscatter during cell death", Ultrasound in Med. \& Biol. 36, 1546-1558 (2010). 
${ }^{4}$ E. Franceschini, R. Guillermin, F. Tourniaire, S. Roffino, E. Lamy, and J.-F. Landrier, "Structure factor model for understanding the measured backscatter coefficients from concentrated cell pellet biophantoms", J. Acoust. Soc. Am. 135, 3620-3631 (2014).

${ }^{5} \mathrm{M}$. Oelze and J. F. Zachary, "Examination of cancer in mouse models using high-frequency quantitative ultrasound", Ultrasound in Med. \& Biol. 32, 1639-1648 (2006).

${ }^{6}$ H. Schmid-Schonbein, G. Gallasch, J. V. Gosen, E. Volger, and H. J. Klose, "Red cell aggregation in blood flow. I. New methods of quantification", Klin. Wschr. 54, 149-157 (1976).

7 H. Schmid-Schonbein, H. Malotta, and F. Striesow, "Erythrocyte aggregation: causes, consequences and methods of assessment", Tijdschr NVKS 15, 88-97 (1990).

8 D. Savery and G. Cloutier, "A point process approach to assess the frequency dependence of ultrasound backscattering by aggregating red blood cells", J. Acoust. Soc. Am. 110, 3252-3262 (2001).

9 F. T. H. Yu, and G. Cloutier, "Experimental ultrasound characterization of red blood cell aggregation using the structure factor size estimator", J. Acoust. Soc. Am. 122, 645-656 (2007).

10 E. Franceschini, B. Metzger, and G. Cloutier, "Forward problem study of an effective medium model for ultrasound blood characterization", IEEE Trans. Ultrason., Ferroelectr., Freq. Control 58, 2668-2679 (2011).

11 E. Franceschini, R. K. Saha, and G. Cloutier, "Comparison of three scattering models for ultrasound blood characterization," IEEE Trans. Ultrason., Ferroelectr., Freq. Control 60, 2321-2334 (2013).

12 P. M. Morse and K. U. Ingard, "Theoretical Acoustics", (Princeton, NJ: Princeton University Press, 1968), Chap. 8, pp. 400-466.

${ }^{13}$ M. F. Insana and D. G. Brown, "Acoustic scattering theory applied to soft biological tissues", in Ultrasonic Scattering in Biological Tissues, edited by K. K. Shung and G. A. Thieme (CRC, Boca Raton, FL, 1993), Chap. 4, pp. 76124.

${ }^{14}$ R. K. Saha, E. Franceschini, and G. Cloutier, "Assessment of accuracy of the structure-factor-size-estimator method in determining red blood cell aggregate size from ultrasound spectral backscatter coefficient," J. Acoust. Soc. Am. 129, 2269-2277 (2011).

15 V. Twersky, "Low-frequency scattering by correlated distributions of randomly oriented particles," J. Acoust. Soc. Am. 81, 1609-1618 (1987).

16 M. S. Wertheim, "Exact solution of the Percus-Yevick integral equation for hard spheres", Physical Review Letters 10, 321-323 (1963).

17 E. Franceschini and G. Cloutier, "Modeling of ultrasound backscattering by aggregating red blood cells", in Quantitative ultrasound in soft tissues, edited by J. Mamou and M. L. Oelze, (Springer, 2013), chap. 6, pp. 117-146.

${ }^{18}$ E. L. Hinrichsen, J. Feder, T. Jossang, "Geometry of random sequential adsorption", Journal of Statistical Physics 44, 793-827 (1986).

19 A. Guinier, G. Fournet, C. B. Walker, and G. H. Vineyard, "Small-Angle Scattering of X-Rays", Physics Today 9 (1955), Chap. 2, pp. 5-82. 
FIGURE CAPTIONS

Figure 1. (Color online) Examples of two cell aggregates of radius $r_{a g} / a=7$, on the left with a compactness of $\phi_{i}=10 \%$, and on the right with a compactness of $\phi_{i}=40 \%$. The radius of the external envelope in displayed with transparency.

Figure 2. (Color online) Differential backscattering cross section of one cell aggregate of radius $r_{\mathrm{ag}} / a=6$ and of compactness $\phi_{i}=0.3$. The symbols represent the $\sigma_{\text {ag,sim }}$ computation. (a) The dashed line (or the solid line, respectively) represents the theoretical coherent component $\sigma_{\text {ag,coh }}$ given in Eq. (11) using $F\left(r_{\mathrm{ag}}\right)$ (or $F\left(\sqrt{5 / 3} R_{\mathrm{g}}\right.$ ), respectively). (b) The solid line (or the dashed line, respectively) represents the theoretical $\sigma_{\text {ag }}$ (or $\sigma_{\mathrm{ag}, \mathrm{G}}$, respectively), both using $F\left(\sqrt{5 / 3} R_{\mathrm{g}}\right)$ in the coherent component.

Figure 3. (Color online) Differential backscattering cross sections of one cell aggregate for different aggregate compactnesses. The symbols represents the $\sigma_{\mathrm{ag}, \mathrm{sim}}$ computation. The solid line (dashed line, respectively) represents the theoretical $\sigma_{\mathrm{ag}}$ using Eqs. (1), (11) and (15), (or the theoretical $\sigma_{\mathrm{ag}, \mathrm{G}}$ using Eqs. (1), (11) and (16), respectively).

Figure 4. (Color online) Averaged $k r_{\mathrm{ag}}$ limits, as a function of the aggregate size ranging for $r_{\text {ag }} / a=5$ to 9 , for three compactnesses $\phi_{i}=10 \%$ (a), $\phi_{i}=25 \%$ (b) and $\phi_{i}=40 \%$ (c). The solid lines correspond to the limits of $\sigma_{\mathrm{ag}, \text { coh }}$ computed using $F\left(\sqrt{5 / 3} R_{\mathrm{g}}\right)$ (crosses), and using $F\left(r_{\mathrm{ag}}\right)$ (squares). The dotted lines correspond to the limits of $\sigma_{\mathrm{ag}}$ (diamonds) and $\sigma_{\mathrm{ag}, \mathrm{G}}$ (circles), both computed using the modification of the radius in $F\left(\sqrt{5 / 3} R_{\mathrm{g}}\right)$. Note that the curves marked with crosses and circles are superimposed in Figs. (a) and (b).

Figure 5. (Color online) Frequency dependent backscatter coefficients for various aggregate sizes and compactnesses. Symbols represent simulation results and lines represent theoretical BSCs computed using Eq. (17). (a) The volume fraction occupied by the cells in the medium is $\phi=16 \%$ and the aggregate compactness is fixed to $\phi_{i}=40 \%$. Radii of aggregates vary from $r_{\mathrm{ag}} / a=5$ to $r_{\mathrm{ag}} / a=9$. (b) The volume fraction occupied by the cells in the medium is $\phi=4 \%$ and the aggregate size is fixed to $r_{\mathrm{ag}} / a=7$. Aggregate compactnesses vary from $\phi_{i}=10 \%$ to $\phi_{i}=40 \%$.

Figure 6. (Color online) (a) Theoretical form factor $F\left(k ; \sqrt{5 / 3} R_{\mathrm{g}}\right)$ and structure factor $S\left(k ; a, \phi_{i}\right)$ computed as a function of the frequency for two compactnesses $\phi=10 \%$ and $\phi_{i}=40 \%$ and aggregate size $r_{\mathrm{ag}} / a=6$. (b) and (c) Theoretical differential backscattering cross sections of one cell aggregate $\sigma_{\mathrm{ag}}$, its coherent component $\sigma_{\mathrm{ag}, \mathrm{coh}}$ and incoherent component $\sigma_{\mathrm{ag}, \text { inc }}$, as a function of frequency. 

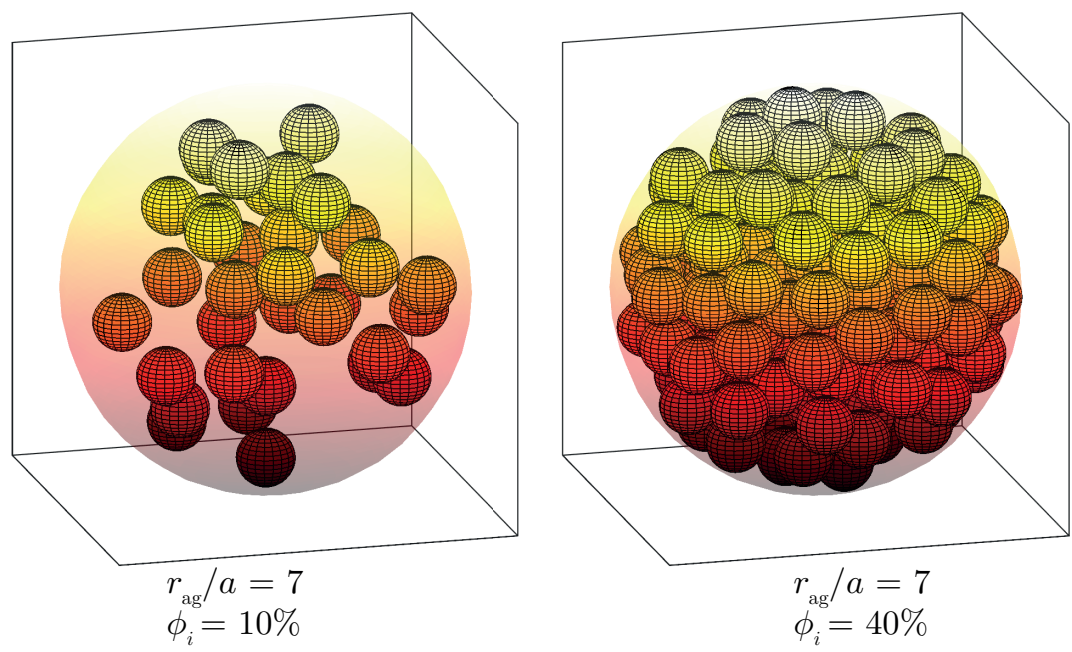

FIG. 1. (Color online) Examples of two cell aggregates of radius $r_{a g} / a=7$, on the left with a compactness of $\phi_{i}=10 \%$, and on the right with a compactness of $\phi_{i}=40 \%$. The radius of the external envelope in displayed with transparency. 


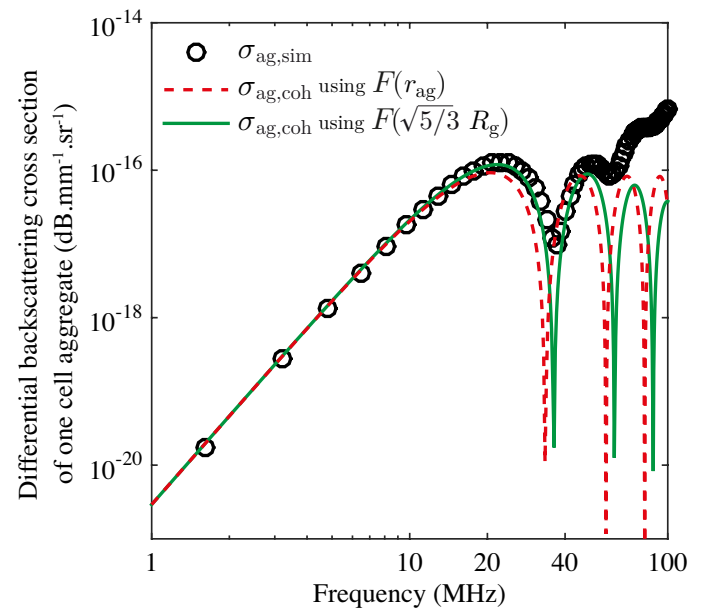

(a)

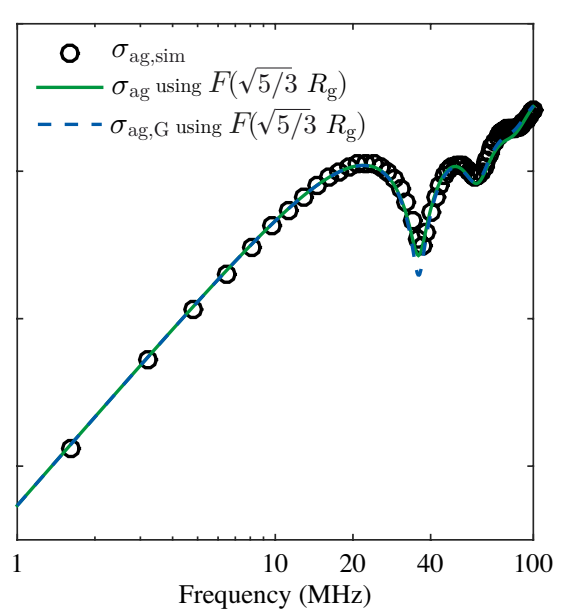

(b)

FIG. 2. (Color online) Differential backscattering cross section of one cell aggregate of radius $r_{\mathrm{ag}} / a=6$ and of compactness $\phi_{i}=0.3$. The symbols represent the $\sigma_{\mathrm{ag}, \mathrm{sim}}$ computation. (a) The dashed line (or the solid line, respectively) represents the

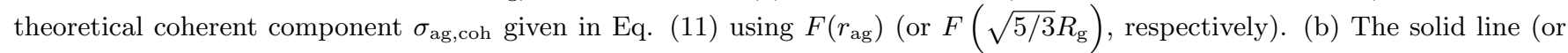
the dashed line, respectively) represents the theoretical $\sigma_{\mathrm{ag}}$ (or $\sigma_{\mathrm{ag}, \mathrm{G}}$, respectively), both using $F\left(\sqrt{5 / 3} R_{\mathrm{g}}\right)$ in the coherent component. 


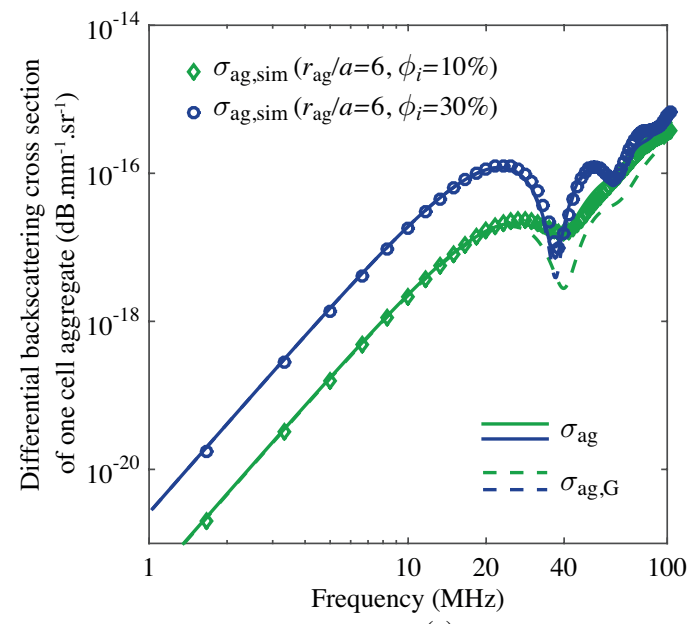

(a)

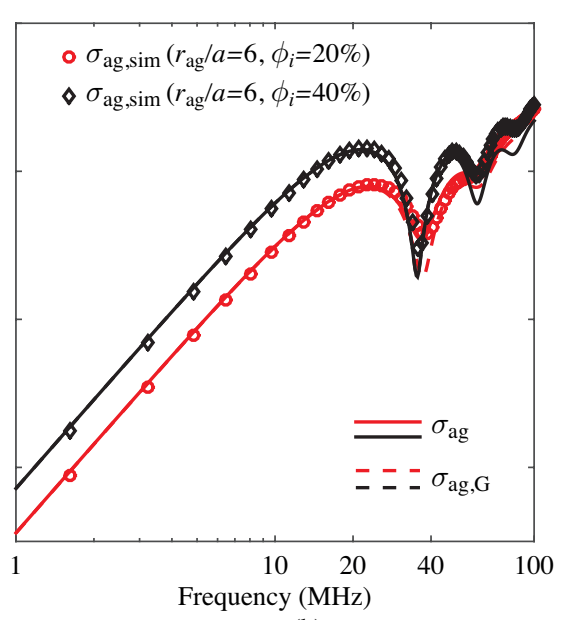

(b)

FIG. 3. (Color online) Differential backscattering cross sections of one cell aggregate for different aggregate compactnesses. The symbols represents the $\sigma_{\text {ag,sim }}$ computation. The solid line (dashed line, respectively) represents the theoretical $\sigma_{\text {ag }}$ using Eqs. (1), (11) and (15), (or the theoretical $\sigma_{\mathrm{ag}, \mathrm{G}}$ using Eqs. (1), (11) and (16), respectively). 

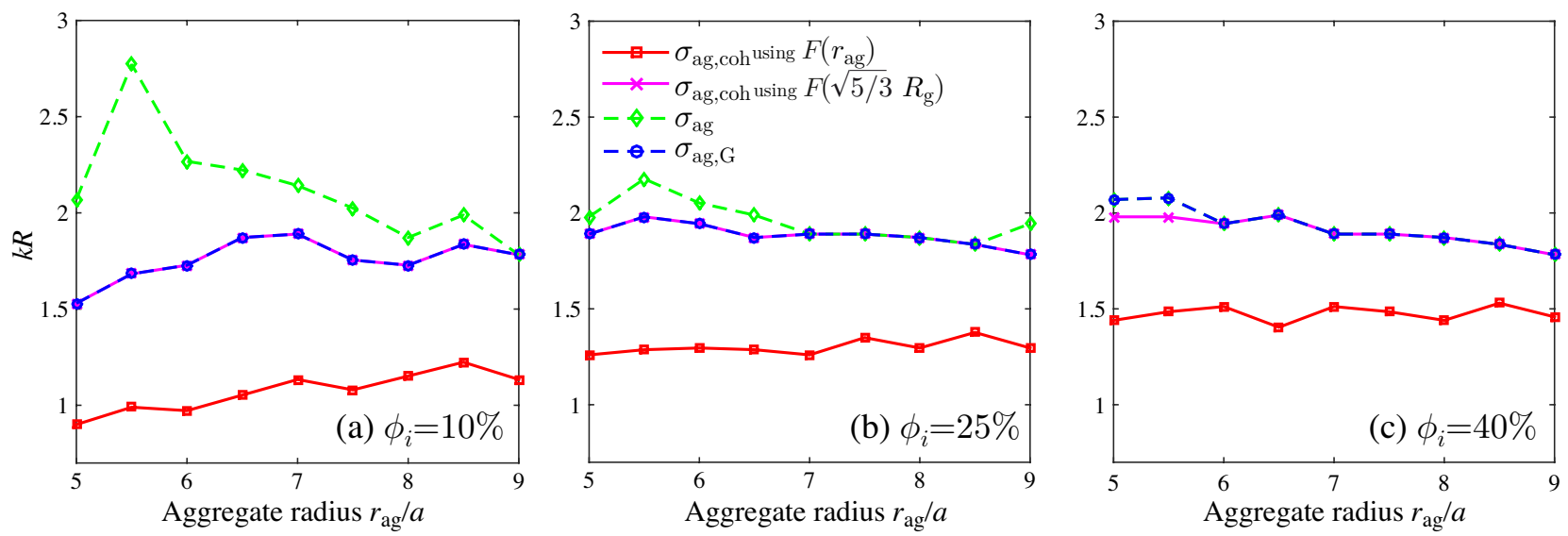

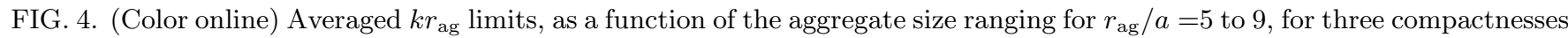
$\phi_{i}=10 \%$ (a), $\phi_{i}=25 \%$ (b) and $\phi_{i}=40 \%$ (c). The solid lines correspond to the limits of $\sigma_{\text {ag,coh }}$ computed using $F\left(\sqrt{5 / 3} R_{\mathrm{g}}\right)$ (crosses), and using $F\left(r_{\mathrm{ag}}\right)$ (squares). The dotted lines correspond to the limits of $\sigma_{\mathrm{ag}}$ (diamonds) and $\sigma_{\mathrm{ag}, \mathrm{G}}$ (circles), both computed using the modification of the radius in $F\left(\sqrt{5 / 3} R_{\mathrm{g}}\right)$. Note that the curves marked with crosses and circles are superimposed in Figs. (a) and (b). 


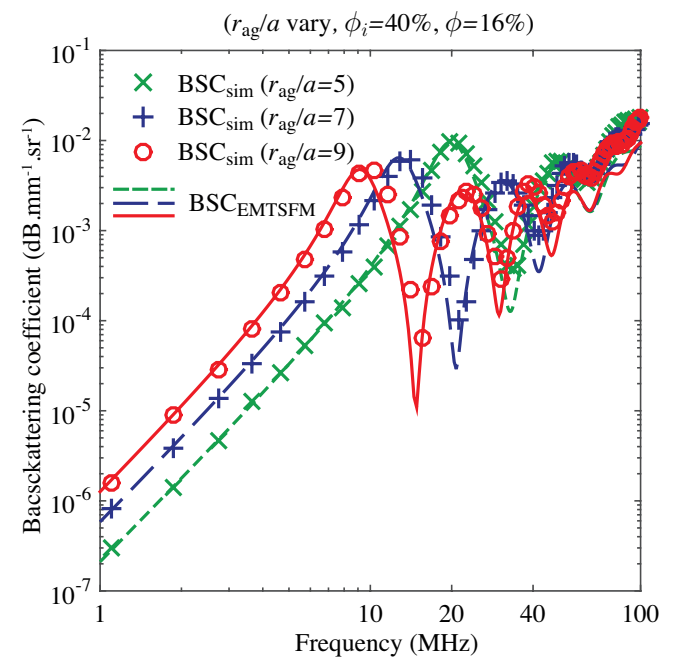

(a)

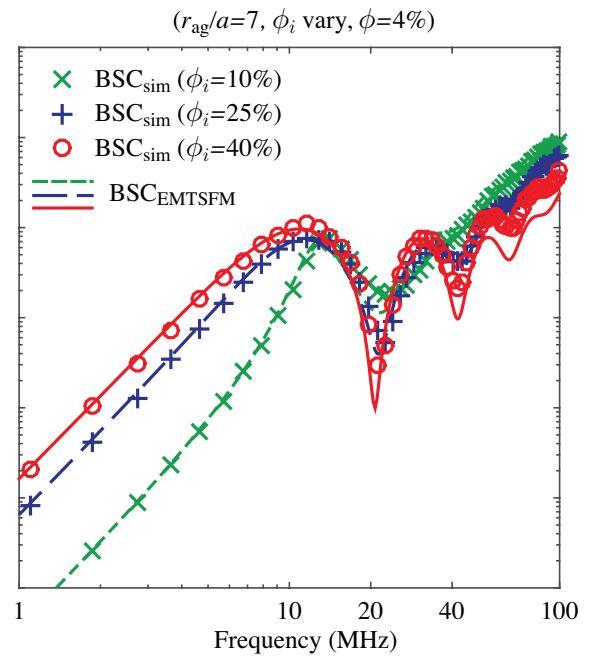

(b)

FIG. 5. (Color online) Frequency dependent backscatter coefficients for various aggregate sizes and compactnesses. Symbols represent simulation results and lines represent theoretical BSCs computed using Eq. (17). (a) The volume fraction occupied by the cells in the medium is $\phi=16 \%$ and the aggregate compactness is fixed to $\phi_{i}=40 \%$. Radii of aggregates vary from $r_{\mathrm{ag}} / a=5$ to $r_{\mathrm{ag}} / a=9$. (b) The volume fraction occupied by the cells in the medium is $\phi=4 \%$ and the aggregate size is fixed to $r_{\mathrm{ag}} / a=7$. Aggregate compactnesses vary from $\phi_{i}=10 \%$ to $\phi_{i}=40 \%$. 


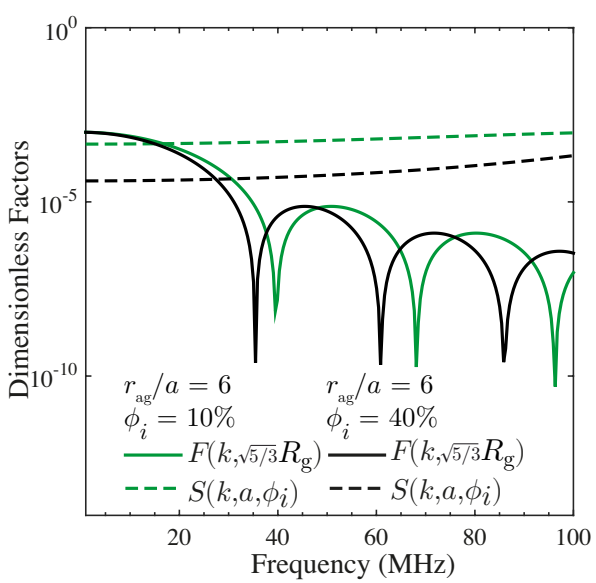

(a)

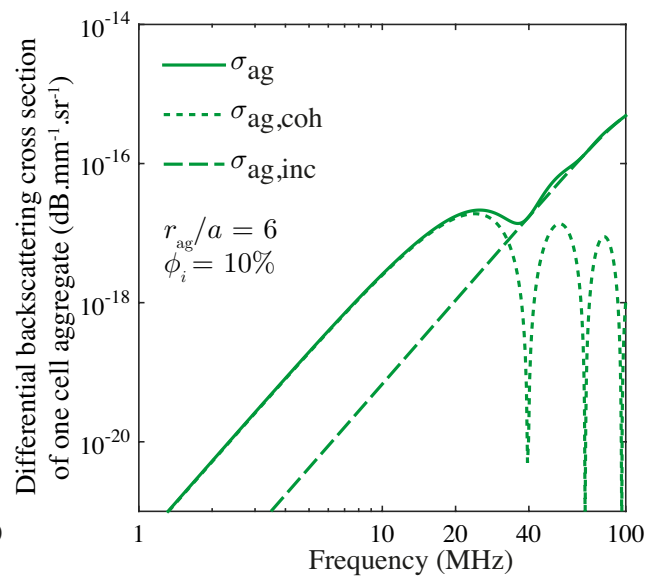

(b)

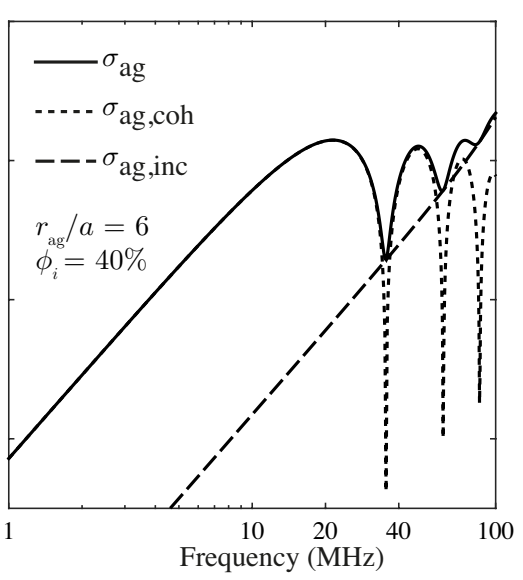

(c)

FIG. 6. (Color online) (a) Theoretical form factor $F\left(k ; \sqrt{5 / 3} R_{\mathrm{g}}\right)$ and structure factor $S\left(k ; a, \phi_{i}\right)$ computed as a function of the frequency for two compactnesses $\phi=10 \%$ and $\phi_{i}=40 \%$ and aggregate size $r_{\mathrm{ag}} / a=6$. (b) and (c) Theoretical differential backscattering cross sections of one cell aggregate $\sigma_{\text {ag }}$, its coherent component $\sigma_{\text {ag,coh }}$ and incoherent component $\sigma_{\text {ag,inc }}$, as a function of frequency. 